\title{
ON THE OPTIMAL CONTROL OF CONTACT-COOKING PROCESSES
}

\author{
Julio R. Banga ${ }^{1, *}$, Zhongli Pan and R. Paul Singh ${ }^{2}$ \\ ${ }^{1}$ Chemical \& Food Engineering Lab, IIM-CSIC, C/Eduardo Cabello 6, 36208 Vigo (Spain) \\ ${ }^{2}$ Dept. of Biological \& Agric. Engineering, University of California, Davis CA 95616 (USA)
}

Paper T01057, accepted in Food \& Bioproducts Processing

Final version, last revised 22 June 2001

\section{SUMMARY}

In this study, we have considered the design of optimised thermal processes for the preparation of cooked hamburger patties. For this purpose, advanced dynamic optimisation (optimal control) techniques have been used. These techniques make use of predictive mathematical models of heat and mass transfer, previously validated with experimental studies. The generic optimal control problem considered was formulated as the determination of the optimal cooking conditions to obtain the highest quality product with an ensured final level of safety in a minimum time. The obtained optimal policies (dynamic heating temperatures) show significant advantages over nominal constant temperature processes. These optimisation studies provide new information to design the next generation of grills with dynamic controls to improve the product quality and safety

KEYWORDS: optimal control, process optimisation, contact-cooking, thermal processing, meat patties

\footnotetext{
* Corresponding author; e-mail: julio@iim.csic.es, ph:+34-986-214473, fax: +34-986-292762
} 


\section{INTRODUCTION}

In the food industry, most processes are operated in batch or semi-continuous mode, so they have an intrinsic dynamic character. In order to calculate the best operating policies, efficient and reliable dynamic optimisation (optimal control) techniques must be used. For example, thermal processing of prepackaged foods, one of the most important preservation techniques, is often carried out in batch retorts. The determination of the optimal retort temperature profile has received considerable attention $^{1,2}$.

In contrast, and despite its great economic importance, the use of dynamic optimisation of cooking still remains in its infancy. A remarkable case involving cooking is that of hamburgers, the fastest growing food items consumed in the United States. According to Balzer $^{3}$, in a 2 week period in 1996, Americans consumed 6 million more hamburgers than the same 2 week period in 1995. U.S. companies benefit from the worldwide popularity of hamburgers, e.g. McDonald's has restaurants in over 100 countries serving several million hamburgers per day. In recent years, the cooking process of hamburger patties has been brought to question due to several outbreaks of food poisoning ${ }^{4-8}$. In the research presented here, hamburguer patties were taken as a case study, useful in developing applications for industrial manufacturing of a wide variety of foods that are non-homogeneous in nature, e.g. sausages, meat balls, and deli meat. In the following, the current state of art on cooking of hamburger patties is described as an illustration of non-homogenous foods.

The primary method of destroying pathogens, such as E. coli O157:H7, in hamburger patties is to cook them to a proper internal temperature. USDA-FSIS ${ }^{9}$ and FDA $^{10}$ recommend that hamburger patties be cooked to an internal temperature of $68.3^{\circ} \mathrm{C}$, with a holding time of $16 \mathrm{~s}$ and $15 \mathrm{~s}$, respectively. Implementation of these standards has been difficult, due to the complexity of measuring the internal temperature in patties and the nonhomogenous composition of hamburger meat. As a result, hamburger patties often are either overcooked, leading to deterioration in textural quality, or undercooked, which presents a potential safety problem. 
A fundamental understanding of the hamburger cooking process and the application of adequate dynamic optimisation techniques can lead to improved specifications and new developments in the design of equipment and sensors that ensure improved safety and quality of cooked patties. To optimise the cooking conditions for achieving improved product quality, predictive models of heat and mass transfer are necessary. The results of predictive heat transfer models can help address food safety issues associated with the survival of pathogens, such as E. coli O157:H7, in undercooked patties ${ }^{11,12}$. Recent outbreaks of E. coli O157:H7 in undercooked hamburger meat (1993 outbreak in U.S., 1996 outbreak in Japan, and another 1996 outbreak in Scotland) emphasise the significance of this study. Also, the use of predictive mathematical modelling can provide valuable insight to the sensitivity of various process conditions.

The overall goal of this research is to design optimized thermal processes for the preparation of cooked hamburger patties using advanced dynamic optimization techniques. These techniques will make use of predictive mathematical models of heat transfer, validated with experimental studies, in order to evaluate by simulation the performance index (i.e, product quality) and constraints. The generic optimal control problem will be formulated as the determination of the optimal cooking conditions to obtain the highest quality product with an ensured level of safety in a minimum time.

\section{RESEARCH OBJECTIVES}

The main objective of this work was to develop, using model-based optimization techniques, improved cooking processes for hamburger patties that assure a safe product with desirable levels of textural quality and yield. The predictive mathematical model used is detailed in Pan et $\mathrm{al}^{13}$. This model describes heat transfer in hamburger patties involving dimensional changes, E. coli O157:H7 destruction, and textural modifications during cooking. 
The optimal cooking operating policies will be computed using dynamic optimization techniques. Due to the highly nonlinear and discontinuous nature of the model, standard gradient-based optimization techniques could not be used, since they failed to converge or converged to local solutions. Thus, and as a second objective of this research, more robust dynamic optimization algorithms, based on stochastic and hybrid (stochasticdeterministic) methods, were developed and implemented.

\section{STATEMENT OF THE OPTIMAL CONTROL PROBLEM}

Here we consider the problem of designing the heating policy of a contact-cooking process in order to obtain maximum patty yield while ensuring the mandatory level of microbiological destruction and final temperature at the coldest point. This can be formulated as an optimization (more precisely, dynamic optimization, or optimal control) problem where our objective is to find the heating temperature profile which gives optimal yield while satisfying the microbial and temperature constraints. The mathematical statement is:

Find the optimal control (heating surface temperature) $T_{\text {heating }}(t)$ over $\mathrm{t} \in\left[0, \mathrm{t}_{\mathrm{f}}\right]$ to maximize the performance index (final patty yield, \%):

$J=y\left(t_{f}\right)=\frac{\bar{m}_{w}+\bar{m}_{f}+1}{m_{w 0}+m_{f 0}+1} \cdot 100$

subject to:

$T_{c}\left(t_{f}\right) \geq T_{r c}$

$N^{*}\left(t_{f}\right) \geq N^{* *}$

$T^{L} \leq T_{\text {heating }}(t) \leq T^{U}$

where Eqn. (1) is the final patty yield, computed from the average final and initial water and fat contents. Variables $\bar{m}_{w}$ and $\bar{m}_{f}$ are the final averaged contents of water and fat 
based on non-fat solids, and are expressed in $\mathrm{kg} / \mathrm{kg}$, while $m_{w 0}$ and $m_{f 0}$ are the values of these variables at initial time. The inequality constraint (2) forces the final temperature at the coldest point, $\mathrm{T}_{\mathrm{c}}\left(\mathrm{t}_{\mathrm{f}}\right)$, to be greater than a required minimum temperature, $\mathrm{T}_{\mathrm{rc}}$. Note that the coldest point is usually taken as the geometric center. Inequality constraint (3) requires the final microbial destruction $\mathrm{N}^{*}\left(\mathrm{t}_{\mathrm{f}}\right)$ to be greater than a required minimum value $\mathrm{N}^{* *}\left(\mathrm{~N}^{*}\right.$ represents the log cycles of microbial destruction, $\mathrm{N}^{*}(\mathrm{t})=\log \left(\mathrm{N}_{0} / \mathrm{N}^{\prime \prime}(\mathrm{t})\right)$, where $\mathrm{N}_{0}$ is the initial average microbial population in the patty, and $N^{\prime \prime}(t)$ is the average population at time $t$, both in CFU/g). The inequality constraints (4) are the upper and lower bounds for the control variable (heating temperature).

There is an additional set of equality constraints which is the process model itself, i.e. the partial differential, ordinary differential and algebraic equations which model the dynamics of the system. The model used here is the one presented in Pan et $\mathrm{al}^{13}$, which describes heat and mass transfer in hamburger patties involving dimensional changes, $E$. coli $\mathrm{O} 157: \mathrm{H} 7$ destruction, and textural modifications during cooking. This model considers all the important factors affecting heat transfer in both frozen and unfrozen patties, namely changes in the thermal properties, cooking losses, ice and fat melting, water evaporation and moving crust. Also, the time-dependent heating surface (grill) temperature and the overall contact heat transfer coefficient between the grill and patty surfaces are handled by appropriate boundary conditions.

The heat transfer process is modeled as one-dimensional (assumption of semi-infinite slab due to the large ratio of diameter to thickness), and it is solved via the enthalpy method $^{11}$ by considering for the effect of mass transfer on enthalpy. The destruction of E. coli $\mathrm{O} 157: \mathrm{H} 7$ at each point inside the domain is modeled by first order kinetics ${ }^{4,12}$, and the average final population is computed using suitable integration procedures. Further details and the complete mathematical statement of the model can be found in $\operatorname{Pan}^{14}$ and Pan et $\mathrm{al}^{13}$. 
It should be noted that other alternative performance indexes (e.g. related with sensorial or nutritional quality) could be easily considered by changing equation (1) and introducing the differential equations reflecting the dynamics (kinetics) of those properties as additional equality constraints. Moreover, several objectives could be considered simultaneously by introducing a multi-objective performance index, e.g. a weighted linear combination where the weights reflect the relative importance given to each objective. Here, we will only present results, without loss of generality, to the case of patty yield maximization.

\section{SOLUTION OF THE OPTIMAL CONTROL PROBLEM}

The optimization problem stated above is a dynamic optimization problem (the term 'dynamic' comes from the differential equations of the process model acting as equality constraints). Dynamic optimization problems are also called optimal control problems or, to be more precise, open loop optimal control problems, since only the initial state of the system is considered to compute the optimal control and no feedback of the states is used during the process. The general optimal control problem (OCP) can be stated as finding the control vector $\underline{u}\{t\}$ and final time $t_{f}$ over $t \in\left[t_{0}, t_{f}\right]$ to minimize (or maximize) a performance index $J[\underline{x}, \underline{u}]$ (where $\underline{x}$ is the vector of state variables) subject to a set of differential-algebraic equality constraints, algebraic inequality constraints and upper and lower bounds for control and state variables. If the process is modeled as a distributed system (which is in fact our case here), the governing partial differential equations are introduced as an additional set of equality constraints.

The dynamic optimization of batch processes is a challenging engineering problem. In the case of food processing, and more specifically in the case of cooking, these problems are especially difficult to solve because of the nonlinear and distributed nature of the system dynamics and the existence of constraints on both the control and state variables. Further, the global optimum might be difficult to achieve due to the insensitivity of the performance index to the control profiles. A number of different techniques have been suggested to solve optimal control problems. These methods can be classified under two main categories: indirect and direct methods. Indirect methods ${ }^{15}$ 
are based on the solution of the necessary conditions (maximum principle of Pontryagin). However, this approach is very difficult in most cases, especially when constraints on the state variables are present. Direct methods transform the original OCP into a nonlinear programming (NLP) problem using complete parameterization ${ }^{16}$ or control parameterization ${ }^{17,18}$. However, many complete and control parameterization strategies rely on deterministic local optimization methods to solve the NLP, so convergence difficulties may appear due to the highly nonlinear and/or discontinuous nature of these systems.

Here we have considered the control vector parameterization (CVP) framework, where the original infinite dimensional optimization problem is transformed into an NLP using a suitable control parameterization scheme, typically using $\mathrm{N}$ control elements of variable size. The process model (set of partial differential, ordinary differential and algebraic equations acting as equality constraints) is solved for each evaluation of the objective function. The resulting NLP, as it has been mentioned, is frequently nonconvex, so standard gradient-based optimization methods might not converge or converge to local solutions. Therefore, methods with global convergence properties should be used. Several deterministic and non-deterministic approaches for global optimization have been proposed ${ }^{19,20}$.

Stochastic methods can be a good alternative to surmount the above mentioned difficulties, as they are usually able to escape from local solutions, locating the vicinity of the global optimum with reasonable computation effort. The ICRS/DS method is an example of adaptive stochastic algorithm which has been successfully used for the solution of several challenging dynamic optimization problems in food processing and biotechnology $\mathrm{y}^{21-24}$ and in chemical engineering ${ }^{20}$.

Genetic algorithms (GAs) are another class of stochastic methods which have become very popular in recent years, with some applications reported in optimal control ${ }^{25}$. Differential Evolution $^{26}$ (DE) is a recent and promising global optimization method partially based on the GA paradigm. Although DE was originally developed for standard (static) optimization, extensions of DE for dynamic optimization have proved 
to be quite efficient as well ${ }^{27,28}$. In fact, Balsa-Canto et $\mathrm{al}^{28}$ compared different stochastic and deterministic strategies, finding that DE performed better than other selected GA-based techniques, and concluding that ICRS/DS and DE were the most efficient and reliable stochastic methods. Therefore, these will be the techniques used in this study.

\section{CASE STUDY}

The cooking of hamburger patties in a double-side clamshell grill is considered. The model parameters used in this study were taken from $\operatorname{Pan}^{14}$ :

Total thickness

$\mathrm{L}=10 \mathrm{~mm}$

Initial patty composition (wb)

Fat: $\mathrm{Y}_{\mathrm{f}}=24 \%$

Water: $\mathrm{Y}_{\mathrm{w}}=60 \%$

Non-fat solids: $16 \%$

Food initial temperature

$$
\mathrm{T}_{0}=-22{ }^{\circ} \mathrm{C}
$$

Mass transfer coefficients Water holding capacity coefficient $\delta_{\mathrm{we}}=0.0132{ }^{\circ} \mathrm{C}^{-1}$

Fat holding capacity coefficient $\delta_{\mathrm{fe}}=0.0159^{\circ} \mathrm{C}^{-1}$

Water transfer coefficient $\mathrm{K}_{\mathrm{w}}=0.015 \mathrm{~s}^{-1}$

Fat transfer coefficient $\mathrm{K}_{\mathrm{f}}=0.017 \mathrm{~s}^{-1}$

Heat transfer coefficient $\quad \mathrm{h}=1200 \mathrm{~W} / \mathrm{m}^{2}{ }^{\circ} \mathrm{C}$

Data tables for other thermophysical properties (enthalpy and thermal conductivity for different temperatures and fat contents) are given in $\operatorname{Pan}^{14}$.

The kinetic parameters for destruction of E. coli $\mathrm{O} 157: \mathrm{H} 7$ were taken as:

$$
\begin{aligned}
& \mathrm{D}_{\mathrm{r}}=5560 \mathrm{~s} \\
& \mathrm{~T}_{\mathrm{r}}=50{ }^{\circ} \mathrm{C} \\
& \mathrm{z}=4.35^{\circ} \mathrm{C} \\
& \mathrm{N}_{0}=10^{6} \mathrm{CFU} / \mathrm{g}
\end{aligned}
$$


The required values for the final temperature and microbiological destruction constraints were:

$$
\begin{aligned}
& \mathrm{T}_{\mathrm{rc}}=68.5^{\circ} \mathrm{C} \\
& \mathrm{N}^{* *}=7.0
\end{aligned}
$$

It should be noted that the above requirement for the final centre temperature is set at final time $t=t_{f}$, which is in fact the end of the heating process. However, because this temperature is quite high, the rate of microbial destruction at $t_{f}$ will be finite, thus microbial destruction will continue during the subsequent cooling of the product (holding time), so the final $\mathrm{N}^{*}$ in the cold product will be larger than that at $\mathrm{t}_{\mathrm{f}}$. This can be regarded as an additional safety factor, so as to robustly cope with possible different cooling conditions.

\section{RESULTS AND DISCUSSION}

In order to establish a reference (or nominal) process to be used for comparisons, we first computed the best (optimal) constant temperature process. This is a simple optimisation problem involving only one decision variable. Considering a total process time of $2 \min \left(t_{f}=120 \mathrm{~s}\right)$, the optimum corresponded to a heating temperature $T(t)=$ constant $=136.2{ }^{\circ} \mathrm{C}$, with an associated performance index (yield) $\mathrm{J}=75.13 \%$. This will be used as a reference value to evaluate optimal controls obtained using different control parameterisations. This best constant temperature process is shown in Figure 1, together with the surface and centre temperatures of the patty. The corresponding curves for microbial destruction and yield are shown in Figures 2 and 3.

Once the reference process was established, the problem of finding optimal (timedependent) heating policies was investigated. Initially, the bounds on the control (heating temperature) were taken as $120 \leq \mathrm{T}(\mathrm{t}) \leq 200{ }^{\circ} \mathrm{C}$. Two types of control parameterisation were considered, piecewise constant (steps) and piecewise linear (ramps), both using $\mathrm{N}$ elements of variable size in order to ensure greater flexibility. A set of optimal control problems was solved considering increasing discretisation levels using the ICRS/DS and DE methods. 
Theoretically, as $\mathrm{N}$ increases, the performance index keeps increasing, approaching the best (truly optimal) performance index $\mathrm{J}$ of the original infinite dimensional problem. However, due to practical considerations, one should choose the process that ensures a near-optimal $\mathbf{J}$ (defined by an admissible tolerance) with a minimum of control elements, as this control profile would be easier to implement in a real process. Considering our particular problem, the results are shown in Table 1.

Clearly, the use of steps is preferable. Besides, for $\mathrm{N}>5$, no significant improvements on $\mathbf{J}$ are achieved, so it can be concluded that the solution for 5 steps, although suboptimal strictly speaking, can be regarded as optimal for all practical purposes (with the additional advantage of ease of implementation). It should be noted that the performance index associated with this optimal control is only marginally better $(\Delta J=$ $2.1 \%)$ than the best constant temperature process. The different optimal controls obtained using steps as control elements are shown in Figure 4, where it can be seen that the control profiles for $\mathrm{N}=4$ and 5 are almost identical, with only very slight differences. These optimal controls are of the bang-bang type, i.e. there are sudden changes in the control from the lower to the upper bound around time $t=82 \mathrm{~s}$, and from the upper back to the lower bound around $\mathrm{t}=100.8 \mathrm{~s}$.

Once the best type and number of control elements was studied, the effect of the lower bound of the control on the final performance index was also investigated, because preliminary computations showed promising results in this direction. Therefore, a set of optimal control problems with different lower bounds was solved, and the results are summarised in Figure 5. It is clear that relaxing this bound improves significantly the final patty yield, achieving $\mathrm{J}=79.33$ for a lower bound of $30{ }^{\circ} \mathrm{C}$, which is a $5.6 \%$ increase of yield with respect to the best constant temperature process. The corresponding optimal control is shown in Figure 6, together with the patty surface and center temperatures. The corresponding plots for microbial destruction and yield are presented in Figures 7 and 8. Once again, there are bang-bang changes in the control. There is an initial heating period with the control at its upper bound until $t=24 \mathrm{~s}$, followed by a period with the control at a temperature around $50{ }^{\circ} \mathrm{C}$ until $65 \mathrm{~s}$, when a 
second maximum temperature period starts. This second period ends by $t=103 \mathrm{~s}$, with the control returning to its lower bound.

These bang-bang control profiles might not be easy to implement in practice using a standard grill because of its thermal inertia, especially considering the relatively small heating times. But those profiles could be implemented using a new design, e.g. a new grill with different heating areas, moving the patty from one to another as needed. In any case, the purpose of this paper was not to consider particular implementation details, but to find out what are the optimal operating policies for this process. This information can then be used to design new units if needed. However, considering a particular existing real unit can be easily done introducing appropriate constraints (e.g. on the heating and cooling rates) to take into account its thermal characteristics.

Regarding the dynamic optimisation methods used, both ICRS/DS and DE solved successfully all the optimal control problems, arriving at very similar results, thus reinforcing the confidence of global optimality. The computation times of both methods were comparable (in the range of 10-15 minutes using a PC Pentium II), although those of DE were usually somewhat larger (up to $30 \%$ ). This is in agreement with the observations of Balsa-Canto et al (1998), although the difference in computational effort reported by these authors was more favourable to ICRS/DS.

In order to illustrate the convergence path followed by these methods, the error curves (relative distance, \%, from the final best solution versus number of evaluations of the performance index) for 3 different runs of each method are shown in Figure 9 (log scale is used in both axes). The paths of DE start after some evaluations because this method, as other GA-based techniques, must compute an initial population before starting. Although there is a certain scattering typical of stochastic methods (the path ultimately depends on a stochastic variable), it is clear that all the trajectories are inside a quite narrow envelope, with no significant differences of convergence rates between DE and ICRS/DS. Also, it can be seen how these methods can arrive at solutions within $1 \%$ of the global optimum in about $300-400$ evaluations, which is very reasonable, especially 
since the methods themselves do not add relevant computational overhead, which is not the case of other (e.g. deterministic) approaches.

\section{CONCLUSIONS AND FUTURE WORK}

Optimal operating policies for contact cooking processes have been obtained by formulating and solving a suitable optimal control problem, making use of validated predictive models. The dynamic optimisation solvers used were based on robust stochastic methods. The obtained optimal policies (dynamic heating temperatures) show significant advantages over nominal constant temperature processes. For the particular case study considered, it has been shown how the final patty yield can be improved up to $6 \%$ with respect to the best nominal process, while satisfying the final temperature and microbial reduction constraints. Variants of this problem considering other objective functions (e.g., energy consumption, controllability requirements, etc.) and constraints will also be studied in the near future. In addition, on-going work is considering the experimental implementation of the optimal operating policies.

These optimisation studies provide new information to design the next generation of grills with dynamic controls to improve the product quality and safety. This information will be useful for food and equipment manufacturers, operators of restaurants and fastfood establishments, consumers, and regulatory agencies for future product development and quality control. Although the focus of this research was on hamburger patties, the information gained is expected to have wide applications in manufacturing prepared foods, a rapidly expanding sector of the U.S. and european food industry.

\section{ACKNOWLEDGEMENTS}

The authors would like to thank NATO for financial support (Collaborative Research Grant CRG-971581). 


\section{REFERENCES}

1 Silva, C. L. M., Oliveira, F. A. R. and Hendrickx, M. (1993) Modeling optimum processing conditions for the sterilization of prepackaged foods. Food Control 1993, 4(2), 67-78.

2 Durance, T. D. (1997) Improving canned food quality with variable retort temperature processes. Trends in Food Sci. \& Technol. 8(4):113-118.

3 Balzer, H. (1996). Eating patterns in America. 11th Annual Report, ND Group, Rosemont, IL

4 Ahmed, N. M., D. E. Conner, and D. L. Huffman. (1995). Heat-resistance of Escherichia Coli O157:H7 in meat and poultry as affected by product composition. Journal of Food Science. 60(3):606-610.

5 Goepfert, J. M. (1977). Aerobic plate count and Escherichia Coli determination on frozen ground-beef patties. Applied and Environmental Microbiology. 34(4):458-460.

6 Hague, M. A., K. E. Warren, M. C. Hunt, D. H. Kropf, C. L. Kastner, S. L. Stroda, and D. E. Johnson. (1994). Endpoint temperature, internal cooked color, and expressible juice color relationships in ground beef patties. Journal of Food Science. 59(3):465-470.

7 Jackson, T. C., M. D. Hardin, and G. R. Acuff. (1996). Heat resistance of Escherichia Coli $\mathrm{O} 157: \mathrm{H} 7$ in a nutrient medium and in ground beef patties as influenced by storage and holding temperatures. Journal of Food Protection. 59(3):230-237.

8 Rita, M., L. Franzetti, A. Mattioli, and A. Galli. (1993). Microorganism lethality during microwave cooking of ground meat. 1. Effect of dishomogeneity of surface power density. Ann. Microbiol. Enzimol. 43:115-129.

9 USDA-FSIS. (1993). Heat processing, cooking, cooling, handling and storage requirements for uncured meat patties. Fed Regist. 58:41138-41152

10 FDA. (1993). Food Code. US Public Health Service, Food and Drug Administration, Washington DC.

11 Singh, R. P. (1996). Heat transfer in beef patties during cooking. Presented at IFT International Annual Meeting. New Orleans, Louisiana.

12 Vijayan, V., Z. Pan, and R. P. Singh. (1997). Modeling heat transfer and destruction of E. Coli O157:H7 during cooking of hamburger patties. Presented at 7 th International Congress of Engineering and Food (ICEF7). Brighton, England. 
13 Pan, Z.; R. P. Singh and T. R. Rumsey (2000) Predictive modeling of contactheating process for cooking a hamburger patty. J. Food Eng. 46:9-19.

14 Pan, Z. (1998) Predictive modeling and optimization of hamburger patty contactcooking processes. Ph:D. Dissertation, University of California, Davis.

15 Bryson, A.E. and Ho Y.C. (1975) "Applied Optimal Control", Hemisphere Pub. Corp., New York.

16 Biegler, L.T. (1984). Solution of dynamic optimization problems by successive quadratic programming and orthogonal collocation. Comput. Chem. Eng. 8, 243-248.

17 Goh, C. J.; Teo, K. L. (1988) Control parameterization: a unified approach to optimal control problems with general constraints. Automatica 24:3.

18 Vassiliadis, V. (1993). Computational solution of dynamic optimization problems with general differential-algebraic constraints. Ph.D. Dissertation, Imperial College, University of London, UK.

19 Floudas, C.A. (1995) "Nonlinear and mixed-integer optimization: fundamentals and applications". Oxford University Press, Oxford.

20 Banga J. R. and Seider W. D. (1996) Global optimization of chemical processes using stochastic algorithms. In "State of the Art in Global Optimization", CA Floudas and PM Pardalos (Eds.), p. 563-583. Kluwer Academic Pub., Dordrecht.

21 Banga, J. R., Perez-Martin, R. I., Gallardo, J. M., and Casares, J. J. (1991) Optimization of the thermal processing of conduction-heated canned foods: study of several objective functions. J. Food Eng. 14:25-51.

22 Banga, J. R. and Singh, R. P. (1994) Optimization of the air drying of foods. J. Food Eng. 23:189-211.

23 Banga, J. R., Alonso A. A. and Singh R. P. (1997) Stochastic dynamic optimization of batch and semi-continuous bioprocesses. Biotechnol. Prog. 13(3):326-335.

24 Banga, J. R., Alonso, A. A., Perez-Martin, R. I., and Singh, R. P. (1994) Optimal control of heat and mass transfer in food and bioproducts processing. Comput. Chem. Eng. 18:S699-S705.

25 Michalewicz Z.; Janikow C.Z.; Krawczyk J.B. (1992) A modified genetic algorithm for optimal control problems. Comput. Math. Appl. 23(12):83-94.

26 Storn, R. and Price, K. (1997) Differential Evolution - a simple and efficient heuristic for global optimization over continuous spaces. J. Global Optim. 11:341-359. 
27 Wang, FS; Chiou, JP. (1997) Optimal control and optimal time location problems of differential-algebraic systems by Differential Evolution. Ind. Eng. Chem. Res. 36(12):5348-5357.

28 Balsa-Canto, E., A.A. Alonso and J.R. Banga (1998) Dynamic optimization of bioprocesses: deterministic and stochastic strategies. ACoFoP IV (Automatic Control of Food and Biological Processes), 21-23 Sept., Göteborg, Sweden. 


\section{TABLES}

\begin{tabular}{l|ccc}
\hline $\begin{array}{l}\text { Type of } \\
\text { elements }\end{array}$ & $\boldsymbol{N}$ & $\boldsymbol{J}$ & $\begin{array}{l}\boldsymbol{\Delta} \boldsymbol{J} \\
(\boldsymbol{\%})\end{array}$ \\
\hline ramps & 2 & 75.70 & 0.7 \\
steps & 2 & 76.06 & 1.2 \\
ramps & 4 & 76.40 & 1.7 \\
steps & 4 & 76.73 & 2.1 \\
ramps & 5 & 76.54 & 1.9 \\
steps & 5 & 76.73 & 2.1 \\
\hline
\end{tabular}

Table 1.- Performance index $(J)$ values obtained for different type (ramps, steps) and number $(\mathrm{N})$ of elements of the control parameterisation $\left(\mathrm{t}_{\mathrm{f}}=120 \mathrm{~s}\right) . \Delta J$ is the relative $(\%)$ increase with respect to the best constant temperature process, which has $\mathrm{J}=75.13$. 


\section{LIST OF FIGURES}

Figure 1.- Best constant heating-temperature process $\left(\mathrm{T}(\mathrm{t})=136.24^{\circ} \mathrm{C}\right)$ for $\mathrm{t}_{\mathrm{f}}=120 \mathrm{~s}$, with an associated performance index (yield) $\mathrm{J}=75.13 \%$. The surface and centre temperatures are also represented. The final centre temperature is 68.5 ${ }^{\circ} \mathrm{C}$.

Figure 2.- Cycles of microbial destruction versus time for the process represented in Figure $1\left(N^{*}(t)=\log \left(N_{0} / N^{\prime \prime}(t)\right)\right.$. The final $N^{*}$ is 8.58 .

Figure 3.- Yield (\%) versus time for the process represented in Figure 1 (yield at final time is $75.13 \%$ ).

Figure 4.- Optimal control profiles obtained using steps as control elements $(\mathrm{N}=2,4,5$; see Table 1).

Figure 5.- Effect of the lower bound of the control (heating temperature) on the final performance index of the optimal process $\left(t_{\mathrm{f}}=120 \mathrm{~s}\right.$, piecewise constant control parameterisation).

Figure 6.- Optimal control (heating temperature) computed with ICRS/DS using piecewise constant control parameterisation (5 elements) and control bounds $30<\mathrm{T}(\mathrm{t})<200^{\circ} \mathrm{C}$. The surface and centre temperatures are also represented. The final centre temperature is $68.5^{\circ} \mathrm{C}$, and the corresponding performance index (yield) is $\mathrm{J}=79.33 \%$.

Figure 7.- Cycles of microbial destruction versus time for the process represented in Figure $6\left(N^{*}(t)=\log \left(N_{0} / N^{\prime \prime}(t)\right)\right.$. The final $N^{*}$ is 9.3 .

Figure 8.- Yield (\%) versus time for the process represented in Figure 6 (final yield is $79.33 \%)$.

Figure 9.- Relative final error (\%) versus number of function evaluations for 3 runs of ICRS/DS (broken lines) and DE/DS (solid lines) 